\title{
19951.7483
}

\section{THE EFFECT OF SIMULATED LOW EARTH ORBIT RADIATION ON POLYIMIDES ( UV DEGRADATION STUDY)}

\author{
John S. Forsy the, Graeme A. George, David J.T. Hill, James H. O'Donnell, \\ Peter J. Pomery and Firas A. Rasoul \\ Polymer Materials and Radiation Group \\ The University of Queensland \\ Queensland, 4072, Australia \\ A BSTRACT
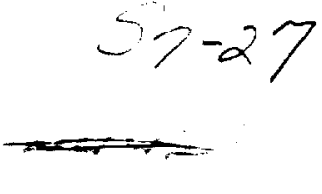 \\ $1-F$
}

\begin{abstract}
UV degradation of polyimide films in air and vacuum were studied using UV-Visible, ESR, FTIR and XPS spectroscopies. The UV-Visible spectra of polyimide films showed a blue shift in the absorption compared to Kapton. This behaviour was attributed to the presence of bulky groups and kinks along the polymer chains which disrupt the formation of a charge transfer complex. The UV-Visible spectra showed also that UV irradiation of polyimides result extensively in surface degradation, leaving the bulk of the polymer intact. ESR spectra of polyimides irradiated in vacuum revealed the formation of stable carbon-centred radicals which give a singlet ESR spectrum, while polyimides irradiated in air produced an asymmetric signal shifted to a lower magnetic field, with a higher g value and line width. This signal was attributed to oxygen-centred radicals of peroxy and/or alkoxy type. The rate of radical formation in air was two fold higher than for vacuum irradiation, and reached a plateau after a short time. This suggests a continuous depletion of radicals on the surface via an ablative degradation process. FTIR, XPS and weight loss studies supported this postulate. An XPS study of the surface indicated a substantial increase in the surface oxidation after irradiation in air. The sharp increase in the $\mathrm{C}-\mathrm{O}$ binding energy peak relative to the $\mathrm{C}-\mathrm{C}$ peak was believed to be associated with an
aromatic ring opening reaction.
\end{abstract}

\section{INTRODUCTION}

Spacecraft in low earth orbit are subjected to significant levels of high energy radiation, including UV and VUV wavelengths. The effects of UV radiation are enhanced over those at the surface of the earth, where the only incident wavelengths are greater than $290 \mathrm{~nm}$ (1). In low earth orbit the incident UV wavelengths extended below $290 \mathrm{~nm}$ into the VUV region, where the Lyman- $\alpha$ emissions of atomic hydrogen occur at $121 \mathrm{~nm}$ (2). In addition to electromagnetic radiation, in low earth orbit polymer materials may also be subjected to atomic oxygen particle radiation, which will result in direct oxidation of the polymer (3)

Thus, polymeric materials for space applications must exhibit a resistance to radiation damage by UV and atomic oxygen. One class of materials which may have this characteristic are the polyimides.

Polyimides are prepared from the reaction of a tetracarboxylic acid dianhydride with a phenylene diamine. Ring opening of the dianhydride readily occurs resulting in the formation of a polyamic acid. The polyamic acid was casted onto a glass plate and cured over night at $300^{\circ} \mathrm{C}$ to achieve maximum imidization (4) 
<smiles>Cc1ccc(Oc2ccc(-n3c(=O)c4cc5c(=O)n(C)c(=O)c5cc4c3=O)cc2)cc1</smiles><smiles>Cc1cccc(-n2c(=O)c3cc4c(=O)n(C)c(=O)c4cc3c2=O)c1</smiles><smiles>Cc1cccc(N2C(=O)c3ccc(-c4ccc5c(c4)C(=O)N(C)C5=O)cc3C2=O)c1</smiles><smiles></smiles><smiles></smiles><smiles>Cc1cccc(Oc2cccc(N3C(=O)c4ccc(Oc5ccc6c(c5)C(=O)N(C)C6=O)cc4C3=O)c2)c1</smiles><smiles></smiles>

Figure 1: The chemical structures of polyimides.

As part of a materials evaluation program for space applications, we have studied the effects of UV induced degradation processes in eight different polyimide films prepared from different dianhydrides and diamines (see Fig 1). Weight loss due to photochemical degradation in an oxidising environment was also determined for comparison with thermal degradation. The study involved the photogeneration of radical species in the polymer 
matrices as the initial steps in the degradation process. In this paper molecular level information for the initial stages of the photodegradation processes obtained from ESR , FTIR and UV-Visible spectroscopies will be discussed. The synergistic effect of UV radiation in air on polyimide surfaces, assessed using $\mathrm{X}$-ray Photoelectron Spectroscopy (XPS) will be also considered.

\section{EXPERIMENTAL}

The polyimide films were exposed to unfiltered UV radiation. The light source used in this study was an Oriel high power mercury/xenon lamp with an output intensity of 9.1 $\mathrm{mW} / \mathrm{cm}^{2}$. The high energy UV wavelength cut off point was about $240 \mathrm{~nm}$. Films irradiated with this lamp were studied using UV/Visible and FTIR spectroscopies, weight loss measurements and XPS spectroscopy.

For the ESR studies, the polyimide films were irradiated in situ in the ESR cavity using a system of lenses and mirrors to focus the radiation on the sample, and a water filter was used to eliminate the infrared radiation which could otherwise cause heating of the sample.

\section{RESULTS AND DISCUSSIONS}

\section{UV-Visible Spectroscopy:}

The UV-Visible spectra of the un-irradiated polyimides showed a large blue shift from the spectrum of Kapton, see Fig 2, with a strong absorption occurring at wavelengths below $400 \mathrm{~nm}$. The reduction in colour of the polyimide films compared to Kapton can be attributed to the effective disruption of the charge transfer complex, giving the polyimides an almost colourless appearance, as in the case of the 6FDA polyimide (5).

It has been recognised that the charge transfer complex governs the colour, thermoplastic flow, dielectric constant and even the UV stability of linear aromatic polyimides (6). In Kapton, both of the imide linkages are attached to the central aromatic ring of the pyromellitic acid, which becomes electron deficient due to the electron withdrawing effect of the four adjacent carbonyl groups. On the other hand, the diamine phenyl rings become electron rich due to electron donating effects of the lone pair of electrons on the nitrogen and the oxygen of the diphenyl ether (ODA) group (7). This relationship causes polarisation and consequently formation of an intramolecular charge transfer complex between the polymer chains $(8,9)$.

In order to reduce the colour of polyimide films, several approaches have been employed to disrupt the formation of the charge transfer complex in those

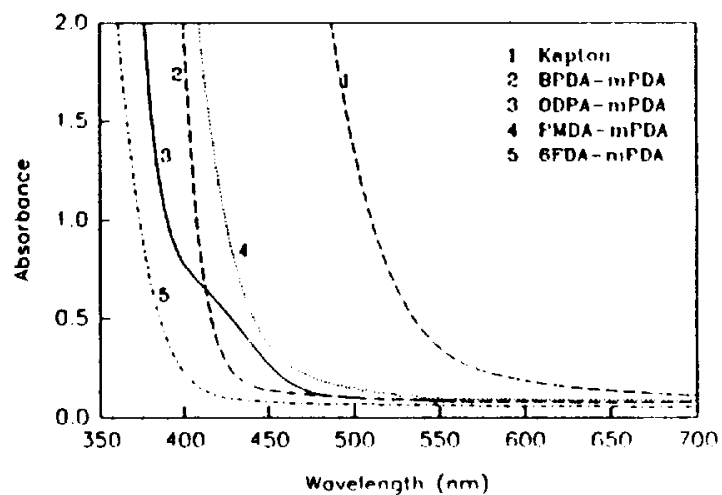

Figure 2: The UV-Visible absorption spectra of polyimides 


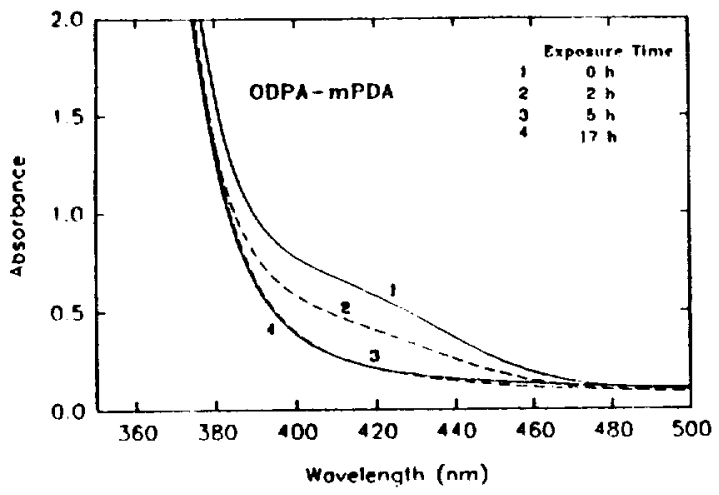

Figure 3: The changes in the UV-Visible absorption spectra with irradiation time of ODPA-mPDA polyimide polymer systems. The use of bulky groups and kinks along the polymer chain are two commonly used approaches by making it physically difficult for the polymer chains to pack close enough to each other to allow the complex to form (10). Furthermore, the introduction of electron withdrawing groups in the dianine and electron donating groups in the dianhydride decreases the charge separation in the polymer, and consequently reduces the possibility of charge transfer complex formation (11). Examples of these approaches are the introduction of oxygen bridges in the dianhydride, as in the ODPA, which

decreases the electron affinity of the polyimides based on this anhydride, the introduction of a bulky group, such as $\mathbf{C F}_{3}$, and the location of the imide groups on different aromatic rings, as in the case of BPDA (see Fig 1). The effects of these strategies is evident in the UV absorption spectrum of PMDA-mPDA polyimide film, for example, which showed a substantial blue shift from Kapton. This behaviour could be explained by the fact that $\mathrm{m}$ phenylene diamine in the PMDA polyimide causes much less polarisation than the ODA in Kapton, despite both imide linkages being attached to the same central aromatic ring (5, 6).

An important feature of the UV-Visible spectra of the polyimide films is their high absorption in the UV region. It has been estimated by Von Sonntag et al. (12) that for the aromatic polyimides the penetration depth of UV radiation is only about $10 \mathrm{~nm}$ for a $95 \%$ reduction of the UV intensity. It could therefore be argued that $U V$ irradiation of these polyimide films would result extensively in surface degradation, leaving the bulk of the material intact.

With the exception of ODPA based polyimides, the UV absorption spectra of the polyimides become slightly red shifted upon exposure to UV radiation in air. This shift may be attributed to the formation of an oxidised species, such as carbonyl groups, on the surface of the films. ODPA polyimides showed a small hump in the visible region of the absorption spectrum before exposure to UV radiation, which could be associated with the oxygen bridge in the dianhydride moiety, (see Fig 3). Interestingly, the hump disappeared after approximately $2 \mathrm{~h}$ of exposure to $\mathrm{UV}$ radiation, then a slight red shift appeared after $5 \mathrm{~h}$ exposure, as observed with the other polyimides. This would indicate that the ODPA polyimides may initially undergo chain scission which disrupts the charge transfer complex, and results in the initial blue shift, then upon further irradiation, the photo-oxidation process causes the red shift through formation of carbonyl groups on the surface.

\section{ESR Studies:}

Samples of the polyimides in quartz tubes were irradiated at room temperature in vacuum and in air using a high power mercury/xenon lamp. 
Typical ESR spectra of the radicals formed on the exposure of the polyimides to UV radiation in vacuum and air are shown in Fig $4 a$ and $4 b$ respectively. The free radical concentration for the polyimides changes substantialky upon UV irradiation in vacuum to produce a symmetrical ESR signal having a $\mathrm{g}$ value of 2.004 and a peak-to-peak line width of $8 \mathrm{G}$. Both the $g$ value and the line width are somewhat larger than those typical of a carbon centred free radical ( $g=2.003$ and $\Delta H_{p p}=3 G$ respectively) (13). The slightly larger $g$ value in the polyimides may be associated with unpaired electrons that are delocalised in the aromatic rings containing nitrogen and oxygen, since the interaction of unpaired electrons with nitrogen and oxygen generally causes the $g$ value to increase, due to their larger spin-orbit coupling constant (14). However, from the ESR spectra alone it is not possible to assign the initial bond cleavage, but it has been postulated by George et al. (13), and later by others (15), that one of the initial photochemical reactions is cleavage of the imide linkage, which contains the comparatively weak $\mathrm{C}-\mathrm{N}$ bond.

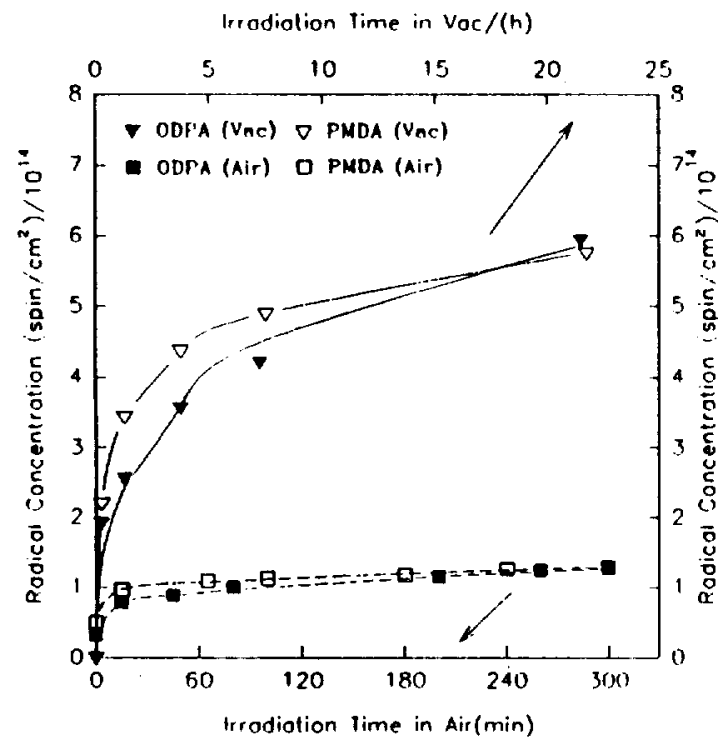

Figure 5: The changes in radical concentration of polyimide films exposed to UV radiation in air and vacuum.
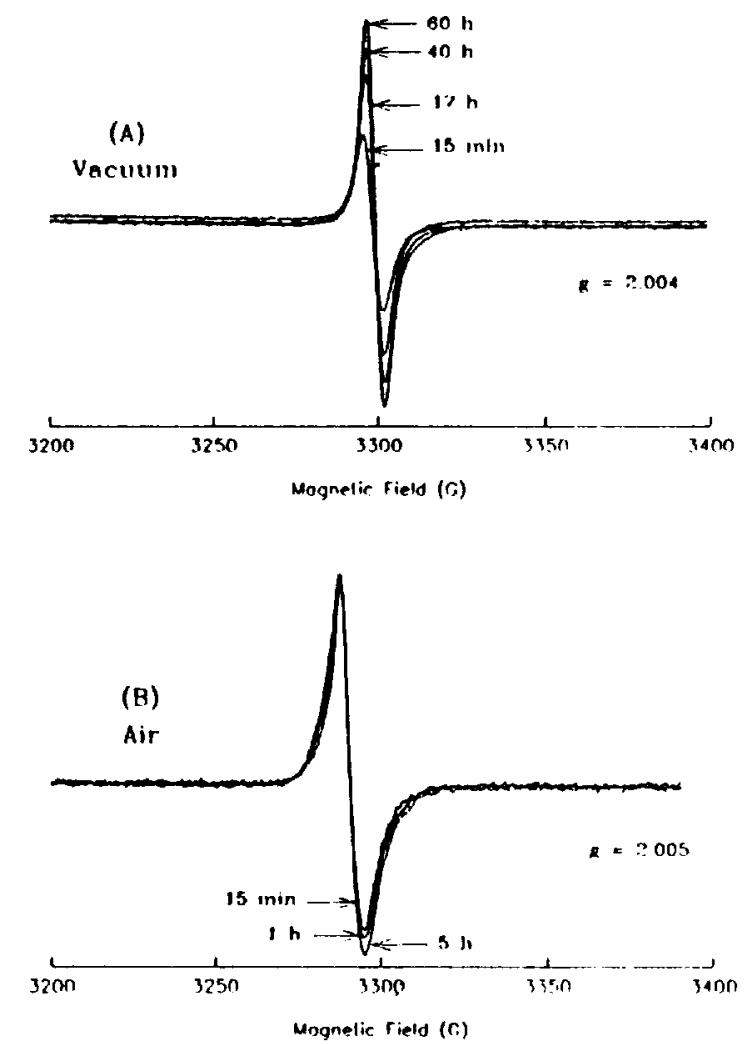

Figure 4: The ESR spectra of BPDA-mPDA polyimide exposed to $U V$ radiation in vacuum (A) and air (B).

The ESR spectra of polyimides irradiated in an oxygen environment are different from those formed for vacuum irradiation. Samples irradiated in air produced an asymmetric ESR signal shifted to a lower magnetic field, with a $\mathrm{g}$ value of 2.005 and a line width of about $14 \mathrm{G}$, see Fig 4b. It was also noticed that the rate of radical formation for polyimides irradiated in the presence of oxygen increased by 2 fold over that for the polymers irradiated in vacuum (see Fig. 5). Moreover, the asymmetric shape of the ESR spectra of polymers irradiated in air suggest that oxygen plays an important role in. the radical formation process, and that oxygen centred radicals contribute to the observed spectrum. Therefore, the ESR spectrum in Fig. 4b could result from radicals being present in both photo-oxidised and 
unphoto-oxidised forms (16). That is, UV irradiation of the polymers in air results in an initial bond cleavage producing radicals on the surface of the films, and these radicals subsequently undergo reaction with oxygen to produce peroxy and alkoxy radicals, which give rise to the oxidised species observed in the UV spectra of the films.

It is wortb mentioning that upon irradiation of Kapton in an oxygen environment, a relatively high concentration of radicals are produced compared with the concentrations observed for the other polyimides; see Fig 6. It is also worth noting that un-irradiated Kapton exhibits a weak asymmetrical ESR signal, with characteristics similar to those found for Kapton following irradiation in an oxygen environment. George et al. (13) claimed that most of the radicals (peroxy and alkoxy) formed by UV irradiation of Kapton in air are located at, or very near, the surface of the polymer, while most of the radicals formed by UV irradiation in vacuum are located at a greater depth in the polymer. This claim was demonstrated by the observed decrease in the ESR signal intensity $(60-70 \%)$ for polymers irradiated in air, followed by soaking of the polymer film in water and or acetone $(13,17)$.

Although the mechanism for formation of these complex surface radicals is unresolved at present, it is clear from the ESR spectra that the radicals observed after irradiation in the presence of oxygen are created at a substantially faster rate than those observed after irradiation in the

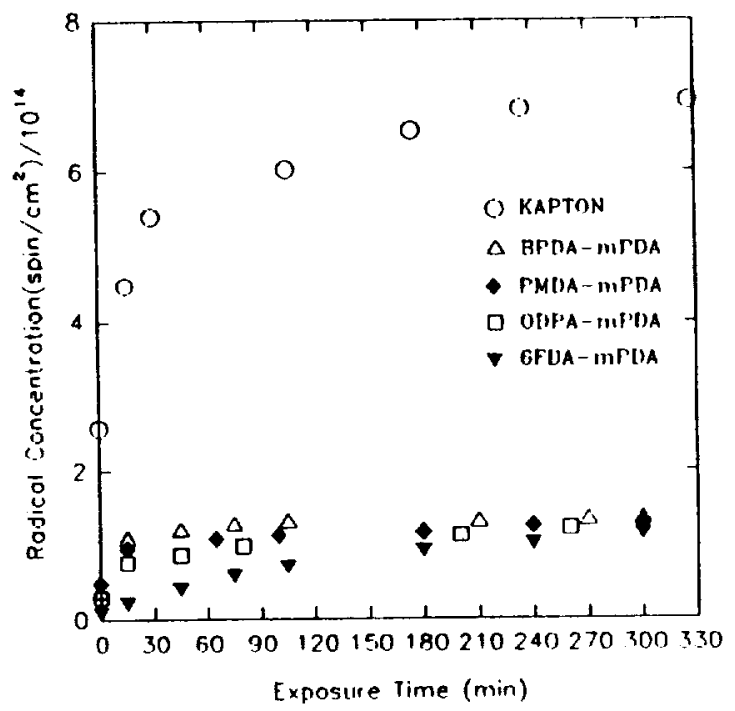

Figure 6: The effect of UV exposure time in air on the radical concentration of Kapton and polyimides. absence of oxygen. Generally, it can be seen from Fig 5 that the radical concentration rapidly reaches a plateau on irradiation in oxygen (after about $1 \mathrm{~h}$ ). These observations could be explained either by the formation of a stable photo-oxidised surface, which prevents further radical formation, or by the depletion of surface radicals via an ablative degradation process.

A transmission FTIR study of the changes in the films on irradiation in air showed that the intensity of all of the absorption peaks decreased with irradiation time, and that no significant new peaks were formed. This observation is consistent with the second of the postulates above. Irradiation in air results in the formation of photo-oxidised species which are lost from the surface by volatilisation. This means that the concentration of the photooxidised species does not build up on the surface, so that no significant absorption from new function groups appears in the IR spectra, and that, as the film surface is eroded away, the film thickness decreases and the intensities of the observed absorption peaks decreases with irradiation time. Hoyle and coworker (18) observed a similar decrease in the intensity of the FTIR absorption spectra of polyimides irradiated in air. These observations are also supported by the observed weight loss in the films with irradiation time. Kapton films exposed to a low earth orbit environment on the leading edge of the 
LDEF showed no new IR absorption peaks, no peaks were missing and that there were no significant shifts in the frequency of various bands $(19,20)$. The films were also eroded by the environment. This is consistent with our observations for UV irradiation in air.

The radical intermediates formed on irradiation are thermally stable and the ESR signal intensity decays only slowly over an extended period of time. One week after irradiation, only $20 \%$ of the radicals have decayed. The slow decay rate might suggest the involvement of various aromatic type radicals, which could be produced either in the absence or the presence of oxygen. This slow radical decay could explain the post irradiation effects observed for polyimide samples after retrieval from LDEF.

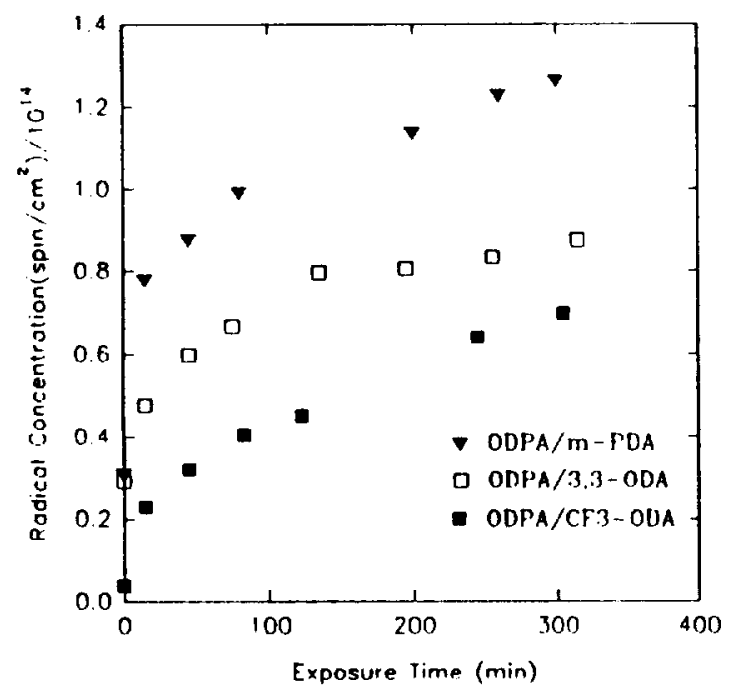

Figure 8: The changes in radical concentration of ODPA polyimides with UV exposure time in air.

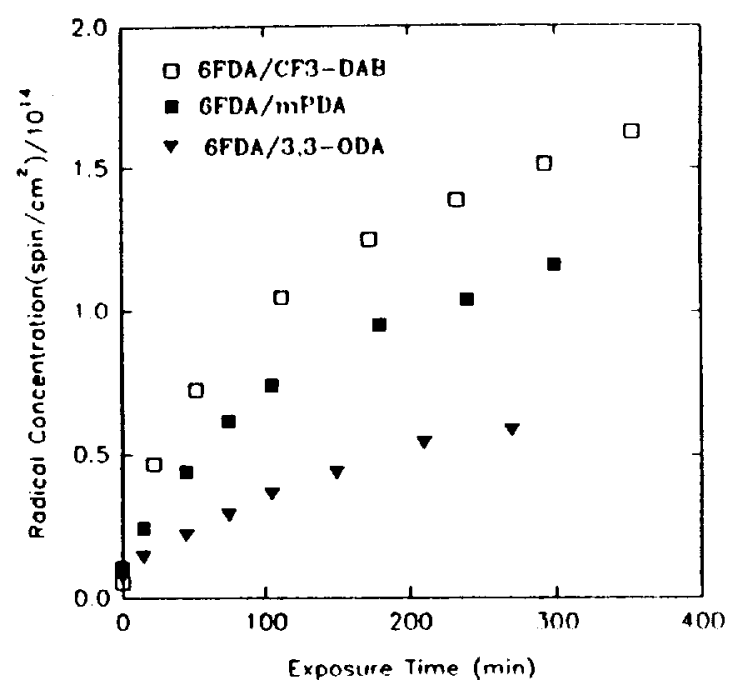

Figure 7 : The changes in radical concentration of 6FDA polyimides with UV exposure time in air.

A simplified free radical chain mechanism for the oxidation of polyimides can be proposed to explain the observed results, in which peroxy and/or alkoxy radicals are formed. These radicals could be formed by direct reaction of oxygen with the radicals produced either on the aromatic rings or at single bonds in the polymer chain (13). The saturation of the radical concentration after long irradiation times could be explained by radical recombination, or by radical loss by volatilisation, in the case of irradiation in the presence of oxygen.

Irradiation of polyimide films in air could provide valuable information on the relative UV stability of polymers compared to Kapton. A close examination of the ESR results of various polyimides revealed that polymers exhibiting a lower radical concentration upon exposure to UV radiation in air are more susceptible to oxidation, and undergo faster erosion and material loss by volatilisation. The build up in the radical concentration with time for two series of polyimides with the same dianhydride and various diamines are shown in Figs $7 \& 8$. These observations are consistent with the behaviour expected for an ablative degradation process. 


\section{Surface Analysis:}

Surface analysis of polyimide films were conducted using XPS spectroscopy. A typical XPS multiplex spectrum (at $25 \mathrm{eV}$ pass energy) for the Cls, Ols and NIs regions of an un-irradiated polyimide film is shown in Fig. 9 for.BPDA-mPDA. The theoretical ratios of $\mathrm{Ols} / \mathrm{Cls}$ and $\mathrm{Nls} / \mathrm{Cls}$ were in agreement with the measured ratios, which suggests that there is no significant contamination of the polymer surface. The $\mathrm{Cls}$ region exhibits two distinct peaks at $285 \mathrm{eV}$ and $288.5 \mathrm{eV}$, which represent the $\mathrm{C}-\mathrm{C}$ plus $\mathrm{C}-\mathrm{H}$ and $\mathrm{C}$ $O$ binding energies respectively. The $\mathrm{C}-\mathrm{N}$ band, which is less sensitive in the XPS analysis than the C-C band, occurs at $285.2 \mathrm{eV}$. It is over shadowed in intensity by the stronger C-C peak. For the fluorinated polyimides, an additional peak arises in the $\mathrm{Cls}$ region for the $\mathrm{C}-\mathrm{F}$ binding energy, which appears at 292.5
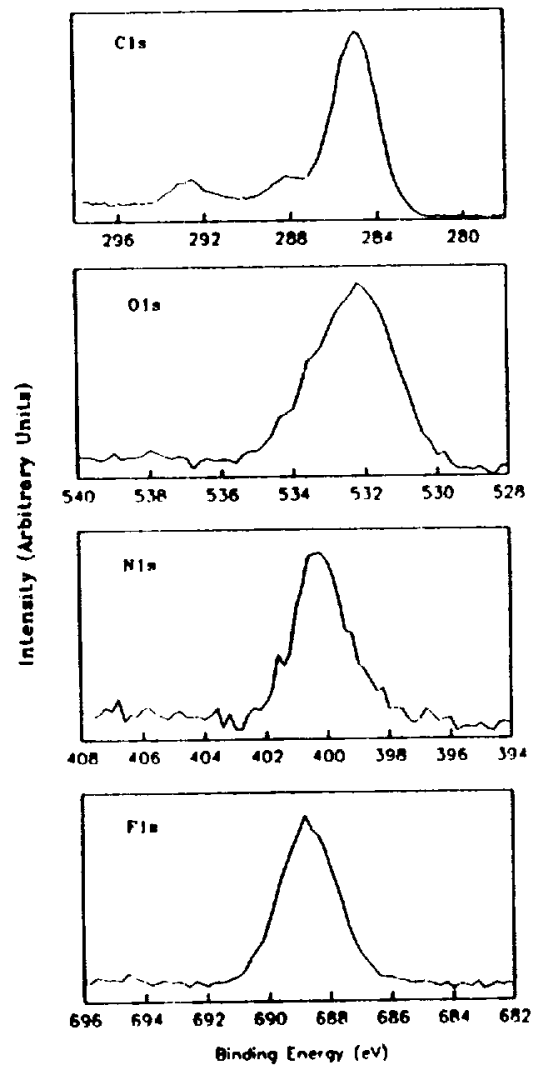

Figuie 10: XPS spectra of unirradiated 6FDA/3,3'ODA polyimide film.
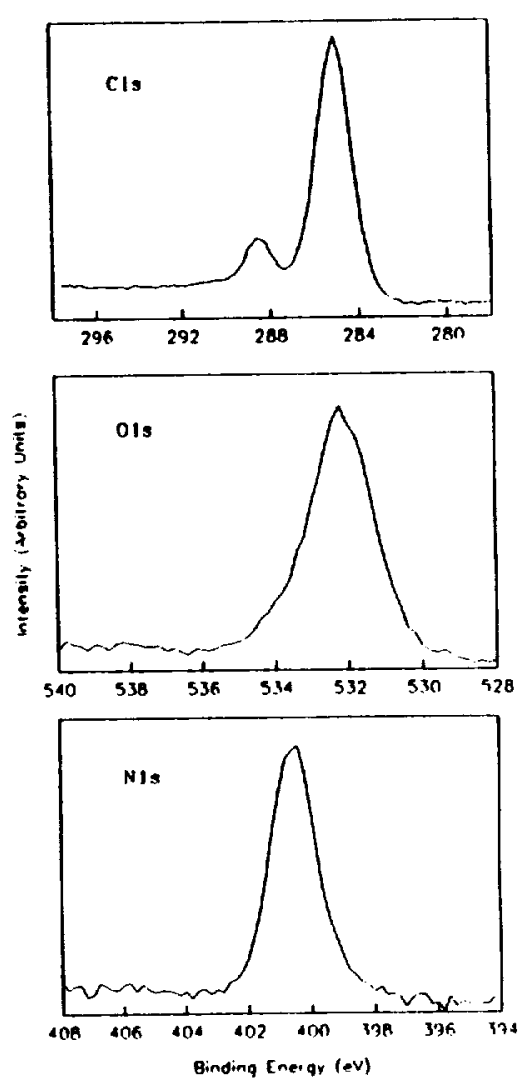

Figuie 9: XPS spectra of unirradiated BPDA-mPDA polyimide film.

eV. Fig 10 shows the Cls, Ols, NIs and Fls regions of the un-irradiated 6FDA-3,3'ODA polyimide.

Exposure of the polyimide films to UV radiation in the presence of oxygen resulted in oxidation of the polymer surface. Figs 11 \& 12 below show the changes in the $\mathrm{Cls}$ and $\mathrm{O}$ ls regions due to exposure to $U V$ radiation in air. It is clear from Fig. 11 that the $\mathrm{Cls}$ region has changed substantially after exposure. The intensity of the $\mathrm{C}-\mathrm{O}$ binding energy peak has increased relative to that of the $\mathrm{C}$-C peak, which indicates an increase in the surface oxidation. Comparing the Ols 
region of the exposed polymer films with the same region of the unexposed polymer, (Fig 12) shows a broadening of the Ols peak, which suggests the presence of different oxidation species on the polymer surface, while the NIs and Fls peaks do not show any significant change. Fig 13 shows a plot of the changes in $01 \mathrm{~s} / \mathrm{Cls}$ ratios with irradiation time for 6FDAmPDA in comparison to Kapton. The ratio of $\mathrm{Ols} / \mathrm{Cls}$ increased sharply after only a few minutes of exposure to UV radiation in air, and then reached a plateau for both polymers after about 30 minutes. This supports the proposal based on the FTIR and the ESR studies that there is continual degradation of the surface during irradiation in the presence of oxygen, producing volatile low molecular weight species. On the other hand, examination of

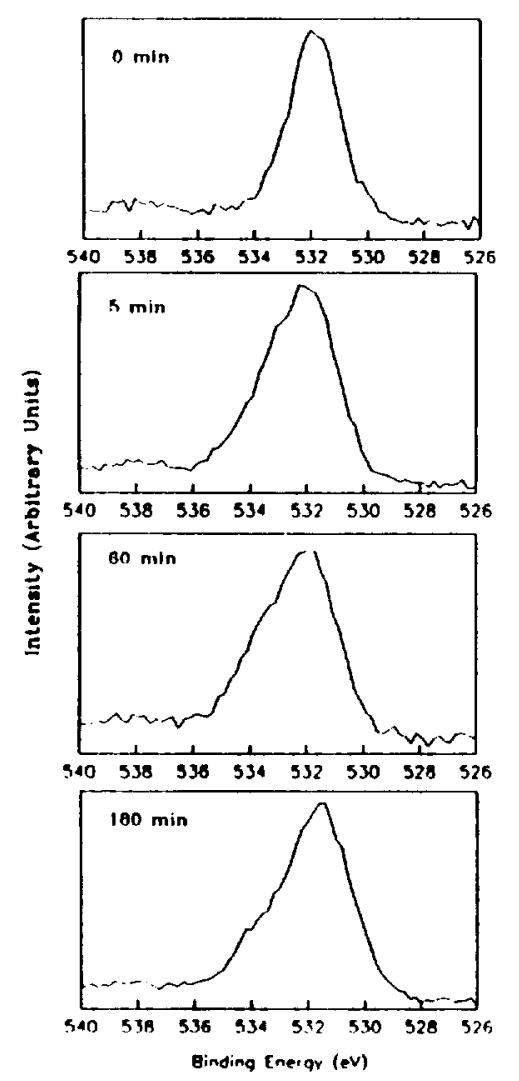

Figure 12: XPS spectra (O1s region) of 6FDA-mPDA polyimide film exposed to $U V$ radiation in air.

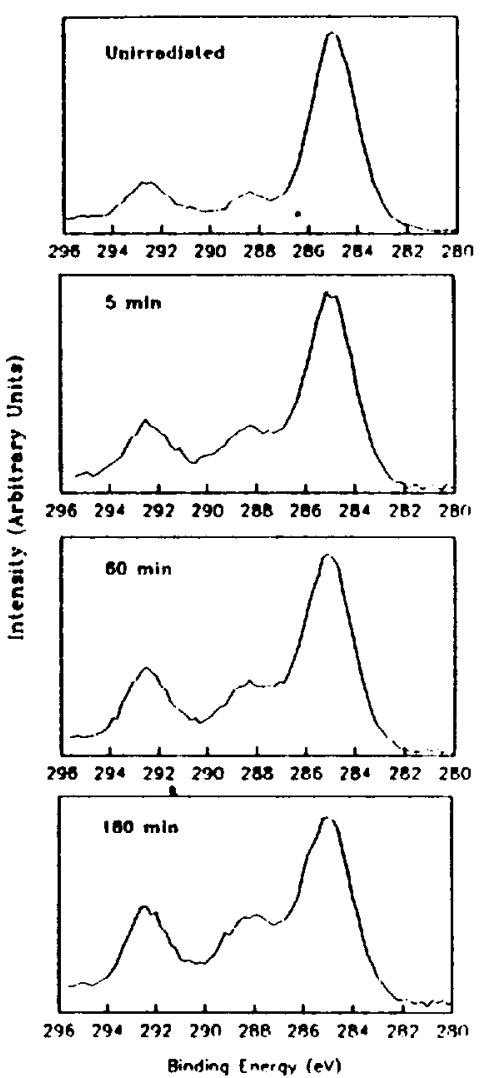

Figure 11: XPS spectra (Cls region) of 6FDA-mPDA polyimide film exposed to $U V$ radiation in air.

the changes in the $\mathrm{Nls} / \mathrm{Cls}$ ratios reveals a concomitant increase in this ratio, which could be related to a reduction in the carbon atom concentration on the polymer surface. This could occur due to surface ablation (21) and loss of low molecular weight oxidised species, such as $\mathrm{CO}$ and $\mathrm{CO}_{2}$, from the polymer surface. Furthermore, it has been reported by Srinivasan et al. (17) that polyimide film exposed to UV radiation for 10 minutes and then soaked for $\mathrm{I} \mathrm{h}$ in water showed a decrease in the $01 \mathrm{~s} / \mathrm{Cls}$ ratio to essentially the value for the un-irradiated polyimide, indicating that the highly photo-oxidised species are effectively removed by dissolution.

The increase in the $01 \mathrm{~s} / \mathrm{Cls}$ ratios on the surface of the polymers can only be accounted for by the opening of the aromatic 
rings during photo-oxidation. Similar aromatic ring opening during photooxidation has been reported for other polymers, including polystyrene (22). These reactions in polystyrene have been reported to involve the complexation of oxygen with surface aromatic groups and the subsequent photolysis of this complex (23). A similar mechanism could apply in the polyimides.

\section{Contact Angle Measuiemients}

Further evidence of surface oxidation was obtained from contact angle measurements. The water/polymer contact angles are sensitive to the surface chemistry and therefore provide information pertaining to the surface chemistry, before and after UV exposure in air. After 15 hours UV

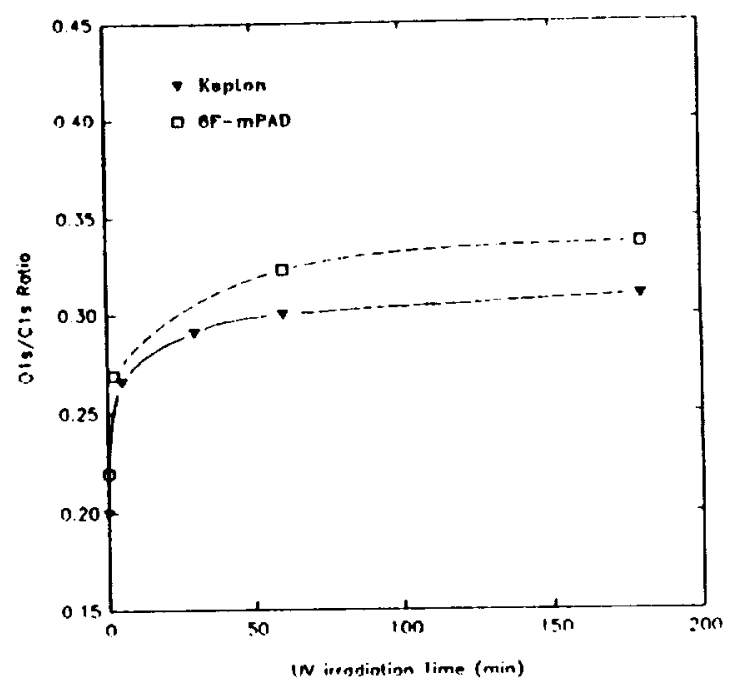

Figure 13: The changes in the $\mathrm{O} / \mathrm{s} / \mathrm{Cls}$ ratio of Kapton and 6FDA-mPDA polyimide exposed to UV radiation in air. exposure, the contact angles for all the polyimides studied, except ODPA/3,3'ODA, have diminished considerably (Table 1), indicating that the surface had become more hydrophilic, and therefore more oxidised.

The water/polymer contact angles were referenced against teflon/water, which agreed well with the literature value of 112 degrees.

It should be noted that ODPA/3,3'-ODA polyimide initially had a small contact angle. This result may be due in part to the absence of fluorine on the surface, although Kapton also lacked fluorine and had a significantly larger contact angle. Errors from inconsistencies in the size of the drop may have been a contributing factor on this result. After irradiation of the ODPA/3,3-ODA polyimide, there was a measured increase in the contact angle. This observation was inconsistent with the other polyimides, and it was thought that surface roughening may have been responsible for this result.

Table 1: Measured water/polyimide contact angles

\begin{tabular}{|l|c|c|}
\hline \multirow{2}{*}{ Polymer } & \multicolumn{2}{|c|}{ Contact Angles (degiees) } \\
\cline { 2 - 3 } & Before Exposure & After Exposure \\
\hline 6FDA/3,3'-ODA & 72 & 54 \\
\hline 6FDA/CF,-DAB & 75 & 46 \\
\hline ODPA/3,3'-ODA & 31 & 35 \\
\hline ODPA/CF,-ODA & 69 & 25 \\
\hline Kapton & 63 & 45 \\
\hline Teflon & 113 & N/A \\
\hline
\end{tabular}




\section{CONCLUSIONS}

In summarising the results of the present investigation, we have shown that polyimides are highly absorbing in the UV region of the spectrum. Therefore, it can be argued that UV irradiation of these polyimides results extensively in surface degradation, leaving the bulk of the polymer unchanged. Furthermore, the stability of polyimides can be associated with their chemical structure and their capacity for forming a charge transfer complex.

The ESR spectra of polyimides irradiated in vacuum reveals the formation of stable radicals which give a singlet ESR spectrum, with a g-value and line width somewhat larger than those typical of carbon-centred free radicals. This suggests that the unpaired electrons are delocalised over the aromatic rings containing nitrogen and oxygen, which are responsible for the slight increase in the g-values due to their larger spin-orbit coupling constant. The primary photodegradation mechanism was believed to involve the cleavage of the imide linkages at the comparatively weak $\mathrm{C}-\mathrm{N}$ bonds. The radical concentration for irradiation in vacuum increased rapidly to a plateau for long exposure times.

The ESR spectra of polyimides irradiated in air produced an asymmetric signal shifted to a lower magnetic field with a higher g-value and a higher line width than that for the polyimides irradiated in vacuum. This ESR signal was attributed to oxygen centred radicals of the peroxy and/or alkoxy type. The rate of radical formation was two fold higher than for vacuum irradiation, and reaches a plateau after only one hour. This suggests a continuous depletion of radicals on the surface via an ablative degradation process. FTIR and weight loss studies supported this postulate. Furthermore, polyimides exhibiting low radical concentration upon irradiation in air are more susceptible to oxygen attack, and have higher rates of degradation than those producing a high radical concentration. This could be due to the fast depletion of radicals from the polymer surface via volatilisation.

Further supporting evidence for the proposed ablation mechanism comes from an XPS study of the surface. The intensity of the $\mathrm{C}-\mathrm{O}$ binding energy peak increased substantially relative to that of $\mathrm{C}-\mathrm{C}$ during irradiation, which indicated an increase in the surface oxidation. Furthermore, the broadening of the Ols peak after exposure to UV radiation in air suggested the presence of different oxidised species on the polymer surface. The initial sharp increase in the $\mathrm{O} 1 \mathrm{~s} / \mathrm{Cls}$ ratio with exposure time, and then the levelling off after about 30 minutes consistent with the postulate of an ablative degradation process. The $\mathrm{N} 1 \mathrm{~s} / \mathrm{C} 1 \mathrm{~s}$ ratio showed a similar behaviour, which could only be related to a reduction in carbon atom concentration on the polymer surface. This can only occur by opening of the aromatic rings and the loss of low molecular weight oxidised species from the polymer surface.

\section{REFERENCES}

1- A.E. Stiegman, D.E. Brinza, M.S. Anderson, T.K. Minton, and E.G. Liang, Jet Propulsion Laboratory Publication, 91-10, California Institute of Technology; Pasadena, California, USA.

2- D. Heath, and M. Thekaekara, 1977, The Solar Output and its Variation, Colorado 
Associated University Press, Boulder, CO, 193.

3- L.E. Murr \& W.H. Kinard, American Scientist, 81, 1993.

4- A.I. Bouise, J. Appl. Polym. Sci., 1986, 32, 4043.

5- A. K. St. Clair and W. S. Slemp, 1991, 23rd International SAMPE Technical Conference.

6- T.L. St. Clair, 1990, in Polyimides, D. Wilson, H.D. Stenzenberger, P.M. Hergenrother, Eds., Blackie Glasgow.

7- R.A. Dine-Hart and W.W. Wright, Makromolek. Chem., 1971, 143, 189.

8- H. Ishida, S.T. Wellinghoff, E. Baer and J.L. Koening, Macromolecules, 1980, 13, 826.

9- E.D. Wachsman, P.S. Martin and C.W. Frank, 1989, in Polymeric Marerials for Electronic Packaging and Interconnection, ACS Symposium Series 407, J.H. Lupinski and R.S. Moore, Eds., Am. Chem. Soc., Washington, D.C., P. 26.

10- A.K. St. Clair, T. L. St. Clair and K. I. Shevket, 1984, Proceedings of the ACS Division of polymeric materials: Science and Engineering vol. 51, p. 62.

11 - A.K. St. Clair \& T.L. St. Clair, 1986, US Pat. 4595548.

12- I.C. Lewis and L.S. Singer, 1981, Chemistry and Physics of Carbon, Marcel Dekker, New York, 17, 1.

13- M. A. George, B.L. Ramakrishna, \& W.S. Glaunsinger, J. Phys. Chem., 1990, 94, 5159.

14- C. Von Sonntag, H.D. Schuchmann, 1977, Adv. Photochem., 10, 6784.

15- T.K. Minton, S.Y. Chung, D.E. Brinza, \& T.A. More, 1993, 3rd LDEF Post Retrieval Symposium Abstracts.

16- B. Ranby and J.F. Rabek, 1977, ESR Spectroscopy in Polymer Research, SpringerVerlag, New York.

17- S. Lazare, P.D. Hoh, J.M. Baker and R. Srinivasan, J. Am. Chem. Soc. 1984, 106, 4288.

18- C.E. Hoyle and E.T. Anzures, J. Appl. Polym. Sci. 1991, 43, 1.

19- P.R. Young, W.S. Slemp and C.R. Gautreux, 1992, 37th International SAMPE, Anaheim, CA, March, 9-12.

20- P.R. Young and W.S. Slemp, 1992, LDEF Materials Workshop 9I, NASA Confernce Publication 3162, part 1, 357.

21- C.E. Hoyle, D. Creed, R. Nagarajan, P. Subramanian and E.T. Anzures, Polymer, 1992, 33, 3162.

22- R.C. Clough, M. Kristiansen and P.R. Ogilby, Macromolecules, 1990, 23, 2698.

23- D.J. Carlsson, R. Brousseau, C. Zhang and D.M. Wiles, Polym. Degrad. Srab., $1987,17,303$.

Acknowledgment: We would like to acknowledge support of this work by the National Aeronautics and Space Administration (NASA), Langley Research Centre. 